\title{
Constraints from orbital motions around the Earth of the environmental fifth-force hypothesis for the OPERA superluminal neutrino phenomenology
}

\author{
L. Iorio \\ Ministero dell'Istruzione, dell'Università e della Ricerca (M.I.U.R.)-Istruzione \\ Fellow of the Royal Astronomical Society (F.R.A.S.) \\ International Institute for Theoretical Physics and Advanced Mathematics Einstein-Galilei \\ Permanent address: Viale Unità di Italia 6870125 Bari (BA), Italy \\ email: lorenzo.iorio@libero.it
}

March 27, 2012

\begin{abstract}
It has been recently suggested by Dvali and Vikman that the superluminal neutrino phenomenology of the OPERA experiment may be due to an environmental feature of the Earth, naturally yielding a long-range fifth force of gravitational origin whose coupling with the neutrino is set by the scale $M_{*}$, in units of reduced Planck mass. Its characteristic length $\lambda$ should not be smaller than one Earth's radius $R_{\mathrm{e}}$, while its upper bound is expected to be slightly smaller than the Earth-Moon distance $\left(60 R_{\mathrm{e}}\right)$. We analytically work out some orbital effects of a Yukawa-type fifth force for a test particle moving in the modified field of a central body. Our results are quite general since they are not restricted to any particular size of $\lambda$; moreover, they are valid for an arbitrary orbital configuration of the particle, i.e. for any value of its eccentricity $e$. We find that the dimensionless strength coupling parameter $\alpha$ is constrained to $|\alpha| \lesssim 1 \times 10^{-10}-4 \times 10^{-9}$ for $1 R_{\mathrm{e}} \leq \lambda \leq 10 R_{\mathrm{e}}$ by the laser data of the Earth's artificial satellite LAGEOS II, corresponding to $M_{*} \gtrsim 4 \times 10^{9}-1.6 \times 10^{10}$. The Moon perigee allows to obtain $|\alpha| \lesssim 3 \times 10^{-11}$ for the Earth-Moon pair in the range $15 R_{\mathrm{e}} \lesssim \lambda \lesssim 60 R_{\mathrm{e}}$, which translates as $M_{*} \gtrsim 3 \times 10^{10}-4.5 \times 10^{10}$. Our results are neither necessarily limited to the superluminal OPERA scenario nor to the Dvali-Vikman model, in which it is $M_{*} \sim 10^{-6}$ at $\lambda \sim 1 R_{\mathrm{e}}$, in contrast with our bounds: they generally extend to any theoretical scenario implying a fifth-force of Yukawa-type.
\end{abstract}

PACS: 04.50.Kd; 96.30.-t; 95.10.Eg; 14.60.Lm; 14.60.St; 13.15.+g

\section{Introduction}

The measured arrival times

$$
\delta t=57.8 \pm\left.\left. 7.8\right|_{\text {stat }}{ }_{-5.9}^{+8.3}\right|_{\text {syst }} \text { ns } \sim 58 \pm 13 \mathrm{~ns}
$$

of the muon neutrinos $\left(\nu_{\mu}\right)$ in the OPERA experiment [1] after a travel along a known baseline distance $d=730 \mathrm{~km}$ from the CERN CNGS beam to the Gran Sasso Laboratory 1 (LNGS)

\footnotetext{
${ }^{1}$ See also the material pertaining to the conference held at CERN on September 2011 here: http://indico.cern.ch/conferenceDisplay.py?confId=155620.
} 
have been interpreted in terms of a property pertaining the motion of the neutrinos themselves. Indeed, it was suggested that their speed $v_{\nu_{\mu}}$ may have overcome the speed of light in vacuum. Stated differently, by defining the dimensionless quantity

$$
\xi \doteq \frac{v_{\nu_{\mu}}-c}{c}
$$

it would be [1]

$$
\xi_{\text {meas }}>0
$$

at a statistical significant level. The result [1]

$$
\xi=\left(2.37 \pm\left.\left. 0.32\right|_{\text {stat }}{ }_{-0.24}^{+0.34}\right|_{\text {syst }}\right) \times 10^{-5} \sim(2.4 \pm 0.5) \times 10^{-5}
$$

was interpreted by Adam et al. [1] by assuming

$$
v_{\nu_{\mu}}^{\text {meas }}=v_{\nu_{\mu}}^{(0)}+\Delta v_{\nu_{\mu}}
$$

with

$$
\Delta v_{\nu_{\mu}}=c\left(\xi_{\text {meas }}+1\right)-v_{\nu_{\mu}}^{(0)}>0,
$$

where $v_{\nu_{\mu}}^{(0)}$ denotes the expected value of the neutrinos' speed: $v_{\nu_{\mu}}^{(0)} \lesssim c$ since the neutrinos have a tiny non-zero mass. It turned out [1] that the measured effect, within the accuracy of the measurement, does not depend on the energy of the muon neutrinos in the domain explored by OPERA (some tens of GeV). Such an interpretation of the neutrinic phenomenology observed in OPERA is unavoidably bound to raise many question, 2 [2, 3, 4, 5, 6. However, [7, 8] pointed out that the statistically significative positive result of eq. (4) might be due to a systematic error of instrumental origin, so that $\xi$ would now be statistically compatible with zero within the error bar. Recently, a time-of-flight measurement of neutrinos from CERN to LNGS performed by the ICARUS collaboration [9] did not confirm the OPERA result by reporting

$$
\delta t=0.3 \pm\left. 4.0\right|_{\text {stat }} \pm\left. 9.0\right|_{\text {syst }} \mathrm{ns} \sim 0.3 \pm 10 \mathrm{~ns},
$$

which is statistically compatible with zero. Note that, as far as the uncertainty in $\xi$ is concerned, eq. (77) naively corresponds to about the same level of eq. (44), i.e. about $0.5 \times 10^{-5}$; indeed, the accuracies in determining $\delta t$ are about the same in both eq. (11) and eq. (17). For earlier studies on superluminal motions of neutrinos in various frameworks, see, e.g., 10, 11, 12, 13, 14, 15, 16,

Dvali and Vikman [17] wondered if the OPERA superluminal phenomenology could be an environmental effect characteristic of the local neighborhood of the Earth, without the need of violation of the Poincaré invariance at a fundamental level. Such a scenario, at an effective field theory level, yields naturally an inevitable appearance of a testable long-range fifth force of gravitational type. Following a clarification by Dvali [18], it is

$$
M_{*} \sim \frac{M_{\mathrm{P}}}{\xi \sqrt{\alpha}}
$$

In it, $M_{\mathrm{P}} \doteq \sqrt{\frac{\hbar c^{5}}{8 \pi G}}=2.43 \times 10^{18} \mathrm{GeV}$ is the energy equivalent of the reduced Planck's mass, where $\hbar$ is the reduced Planck constant, $c$ is the speed of light in vacuum and $G$ is the Newtonian

\footnotetext{
${ }^{2}$ In view of the expected forthcoming large amount of papers dealing with [1, we will not try to formally cite them here because such a list would likely become out-of-date very quickly. See the electronic databases like ArXiv, NASA/ADS, SPIRES/HEP.
} 
constant of gravitation, and the scale $M_{*}$ sets the strength of the coupling of the putative new massive spin-2 degree of freedom to the neutrino [17]. For other investigations involving various aspects of gravitation, astrophysics and cosmology, see, e.g., [19, 20, 21, 22, 23, 24, 25, 26, 27, 28, 29, 30, 31. According to Dvali [18, the range length $\lambda$ should not be shorter then the terrestrial radius $R_{\mathrm{e}}$ : see also [32, in which Earth-size extra dimensions were studies. Data from solar neutrinos, not yet analyzed in this respect, would allow to obtain an upper bound on $\lambda$ which would likely be shorter than the Earth-Sun distance [18. Thus, Dvali [18] argues that $\lambda$ should be something less than the Earth-Moon distance, and larger than Earth's radius.

In this paper, we will explore such an intriguing possibility by analytically working out some orbital effects of a gravitational long-range fifth force of Yukawa-type. We will perform a first-order perturbative calculation without making any a-priori assumptions either on the size of $\lambda$ or on the trajectory's configuration of the test particle orbiting the central body acting as source of the putative exotic effect. We will also put constraints on the strength parameter $\alpha$ for various values of $\lambda$ within the ranges envisaged by Dvali and Vikman [17] in view of the latest results from the orbital determination of some natural and artificial bodies around the Earth. Thus, it will be possible to infer lower bounds on the coupling of neutrino to the new hypothetical force, which is given by $M_{\mathrm{P}} / M_{*}$ [17. The upper bound on $M_{\mathrm{P}} / M_{*}$ comes from astrophysical and cosmological observations like star cooling and Big-Bang Nucleosynthesis (BBN) [32]: a: 3 Dvali and Vikman have shown [17, the requirement that new particle is not affecting star-cooling and BBN yields $M_{*} / M_{\mathrm{P}}>4 \times 10^{-12}-10^{-11}$ or so, corresponding to $M_{*}>10^{7}-10^{8} \mathrm{GeV}$ in equivalent energy units.

The plan of the paper is as follows. In Section 2 we analytically work out some longterm orbital effects due to a Yukawa-like modification of the Newtonian inverse-square law. In Section 3.1 and Section 3.2 we phenomenologically constrain $\alpha$ with the laser data of the Earth's artificial satellite LAGEOS II and the Moon, respectively. We also infer corresponding bounds on $M_{*} / M_{\mathrm{P}}$. Section 4 summarizes our findings.

\section{Analytical calculation of some orbital effects induced by a Yukawa-like fifth force}

The Yukawa-type correction to the usual Newtonian gravitational potential $U_{\mathrm{N}}=-\mu / r$, where $\mu \doteq G M$ is the gravitational parameter of the central body of mass $M$ which acts as source of the supposedly modified gravitational field, is

$$
\Delta U_{\mathrm{Y}}=-\frac{\alpha \mu_{\infty}}{r} \exp \left(-\frac{r}{\lambda}\right)
$$

where $\mu_{\infty}$ is the gravitational parameter evaluated at distances $r$ much larger than the scale length $\lambda$. The total acceleration resulting from

$$
U_{\mathrm{tot}}=U_{\mathrm{N}}+\Delta U_{\mathrm{Y}}=-\frac{\mu_{\infty}}{r}\left[1+\alpha \exp \left(-\frac{r}{\lambda}\right)\right]
$$

is, thus,

$$
A_{\mathrm{tot}}=-\frac{\mu_{\infty}}{r^{2}}\left[1+\alpha\left(1+\frac{r}{\lambda}\right) \exp \left(-\frac{r}{\lambda}\right)\right]
$$

It should be noticed that Dvali and Vikman [17] leave room, in principle, for a compositiondependent fifth-force, so that $\alpha$ may not be the same for different bodies. From eq. (111) the

\footnotetext{
${ }^{3}$ Dvali and Vikman [17] used the reduced Planck units, in which $M_{\mathrm{P}}=1$, and $c=1$.
} 
following considerations can be traced about the relation between $\mu_{\infty}$ and the values $\mu_{\text {meas }}$ of the gravitational parameter actually measured in, e.g., ranging experiments to terrestrial artificial and natural satellites, interplanetary probes and planets themselves. Indeed, since the Yukawa-like corrections to the Newtonian accelerations felt by the test particles are usually not included in the dynamical force models fit to the observations, an "effective" value of the gravitational parameter is, actually, measured, i.e. it is

$$
\mu_{\text {meas }}=\mu_{\infty}\left[1+\alpha\left(1+\frac{r}{\lambda}\right) \exp \left(-\frac{r}{\lambda}\right)\right] .
$$

This implies that

$$
\left\{\begin{aligned}
\mu_{\text {meas }} & \approx \mu_{\infty}, r \gg \lambda, \\
\mu_{\text {meas }} & =\mu_{\infty}[1+2 \alpha \exp (-1)], r=\lambda, \\
\mu_{\text {meas }} & \approx \mu_{\infty}(1+\alpha), r \ll \lambda .
\end{aligned}\right.
$$

Since $\alpha$ is, of course, expected to be quite small, it is reasonable and adequate to assume

$$
\mu_{\infty} \approx \mu_{\text {meas }}
$$

also for $r \lesssim \lambda$ in practical calculations of the perturbative effects of eq. (9) (see eq. (16) below); our further analysis will show a-posteriori that this is just the case, given the upper bounds on $\alpha$ which will be inferred. Strictly speaking, the use of the measured values $\mu_{\text {meas }}$ in those places in the formulas in which $\mu_{\infty}$ appears would be justified only if $\lambda$ was much smaller than $r$ : this would be a fatal restriction because, e.g., $\mu_{\odot}$ is routinely measured from interplanetary ranging mainly involving the inner planets of the solar system, i.e. one would be forced to consider only the case $\lambda \ll 0.38 \mathrm{au}=6 \times 10^{10} \mathrm{~m}$.

In view of a first-order perturbative calculation, it is, now, useful to evaluate $\Delta U_{\mathrm{Y}}$ onto the unperturbed Keplerian ellipse and average it over one orbital revolution of the test particle. By using the eccentric anomaly $E$ as fast variable, the result is

$$
\left\langle\Delta U_{\mathrm{Y}}\right\rangle=-\frac{\alpha \mu_{\infty} \exp \left(-\frac{a}{\lambda}\right)}{a} I_{0}\left(\frac{a e}{\lambda}\right)
$$

where $a, e$ are the semimajor axis and the eccentricity, respectively, of the orbit of the test particle, and $I_{0}(x)$ is the modified Bessel function of the first kind $I_{k}(x)$ for $k=0$. Note that eq. (15) is an exact result: no approximations have been used either for the orbital configuration of the test particle or the size of the scale parameter $\lambda$. From eq. (15) it is possible to obtain perturbatively the secular precessions of both the pericenter $\omega$ and the mean anomaly $\mathcal{M}$ by using the Lagrange planetary equations 33 .

Concerning $\omega$, we have

$$
\left\langle\dot{\omega}_{\mathrm{Y}}\right\rangle=\alpha \sqrt{\frac{\mu_{\infty}\left(1-e^{2}\right)}{a}} \frac{\exp \left(-\frac{a}{\lambda}\right)}{e \lambda} I_{1}\left(\frac{a e}{\lambda}\right),
$$

where $I_{1}(x)$ is the modified Bessel function of the first kind $5 I_{k}(x)$ for $k=1$. Notice that eq. (16) agrees with the result obtained by [34] with a different approach. More specifically, 34]

\footnotetext{
${ }^{4}$ See on the WEB http://mathworld.wolfram.com/ModifiedBesselFunctionoftheFirstKind.html and references therein.

${ }^{5}$ See on the WEB http://mathworld.wolfram.com/ModifiedBesselFunctionoftheFirstKind.html and references therein.
} 
worked out the Yukawa-like pericenter advance per orbit: it can straightforwardly be obtained from eq. (16) by taking the product of $\left\langle\dot{\omega}_{\mathrm{Y}}\right\rangle$ times the orbital period $P_{\mathrm{b}} \doteq 2 \pi / n=2 \pi \sqrt{a^{3} / \mu_{\infty}}$. The precession of eq. (16) loses its meaning for $e \rightarrow 0$ since it yields $0 / 0$. Other derivations of either the Yukawa-type secular precession of the pericenter or its advance per orbit can be found in, e.g., [35, 36, 37, 38, 39, 40, 41, 42, 43, 44, 45, 46, 47, 48, 49, 50, 51, 52, 53, 54. All of them make use of different level of approximations in either the magnitude of the length scale $\lambda$ or the orbital configuration of the test particle. From eq. (16) it is possible to infer

$$
|\alpha| \leq \delta(\Delta \dot{\varpi}) \sqrt{\frac{a}{\mu_{\infty}\left(1-e^{2}\right)}} \frac{e \lambda \exp \left(\frac{a}{\lambda}\right)}{I_{1}\left(\frac{a e}{\lambda}\right)},
$$

where $\delta(\Delta \dot{\varpi})$ can be thought as the uncertainty in some observationally determined correction $\Delta \dot{\varpi}$ to the standard secular precession of the pericenter for some astronomical system. We will use eq. (17) in Section 3.1 and Section 3.2 for the geodetic satellite LAGEOS II and the Moon, respectively.

\section{Constraints from laser-ranging}

Let us start to consider the range $10^{6}-10^{7} \mathrm{~m}$ corresponding to approximately $1-10$ Earth's radii $R_{\mathrm{e}}$. The traditional constraints on $\alpha$ for $\lambda$ lying in the aforementioned range are depicted in Figure 1 of [55] or Figure 4 of [39], based on Figure 2.13 of [36] and adapted by [56] in his Figure 4, Figure 1 of [35], and Figure 1 of [57]: they are of the order of $10^{-5}-5 \times 10^{-8}$. The technique with which they have been obtained is described in detail in [36]. It is based on the determination of the Earth's gravitational parameter $\mu_{\mathrm{e}}$ from laser-ranging measurements at the altitudes of the LAGEOS satellite and the Moon, and on ground-based measurements of the terrestrial gravitational acceleration.

More specifically, the LAGEOS-Moon constraint of the order of $\approx 5 \times 10^{-8}$ is obtained in the following way. First, the ratio

$$
\eta_{\mathrm{Y}}^{\prime} \doteq 2\left[\frac{r_{\mathrm{L}}^{2} A\left(r_{\mathrm{L}}\right)-r_{\mathrm{M}}^{2} A\left(r_{\mathrm{M}}\right)}{r_{\mathrm{L}}^{2} A\left(r_{\mathrm{L}}\right)+r_{\mathrm{M}}^{2} A\left(r_{\mathrm{M}}\right)}\right],
$$

where6

$$
A\left(r_{\mathrm{L} / \mathrm{M}}\right)=-\frac{\mu_{\infty}}{r_{\mathrm{L} / \mathrm{M}}^{2}}\left[1+\alpha\left(1+\frac{r_{\mathrm{L} / \mathrm{M}}}{\lambda}\right) \exp \left(-\frac{r_{\mathrm{L} / \mathrm{M}}}{\lambda}\right)\right]
$$

denotes the Newtonian+Yukawa acceleration, to be evaluated at distances $r_{\mathrm{L} / \mathrm{M}}$, is theoretically computed: eq. (18) is, by construction, independent of $\mu_{\infty}$. Then, it is compared to the analogous ratio $\eta_{\mathrm{N}}^{\prime}$ computed for the empirically determined values $\mu_{\text {meas }}^{(\mathrm{SLR})}$ and $\mu_{\text {meas }}^{(\mathrm{LLR})}$ of the Earth's gravitational parameter at $r_{\mathrm{L}}$ and $r_{\mathrm{M}}$ from Satellite Laser Ranging (SLR) and Lunar Laser Ranging (LLR) measurements divided by the square of the Earth's radius $R_{\mathrm{e}}$ : in the computation of $\eta_{\text {meas }}^{\prime}$ we assume Newtonian values for the accelerations $A\left(r_{\mathrm{L} / \mathrm{M}}\right)$. Basically, $\eta^{\prime}$ is the difference between the values of $\mu_{\mathrm{e}}$ evaluated at two different distances normalized to the average of such two values: in the Newtonian dynamics it vanishes, while in the framework of the Yukawa-like deviations from the Newtonian picture $\eta^{\prime}$ is different from zero. The comparison between such two determinations of $\eta^{\prime}$ yields upper bounds on $\alpha$ for various values of $\lambda$.

\footnotetext{
${ }^{6}$ Here $r_{\mathrm{L} / \mathrm{M}}$ is a shorthand for designing alternatively either $r_{\mathrm{L}}$ or $r_{\mathrm{M}}$.
} 
Instead, the LAGEOS-Earth constraint of about $10^{-5}$ comes from a comparison between the empirical ratio

$$
\eta_{\mathrm{N}} \doteq \frac{A_{\text {terr }}\left(R_{\mathrm{e}}\right)-A_{\mathrm{L}}\left(R_{\mathrm{e}}\right)}{A_{\mathrm{L}}\left(R_{\mathrm{e}}\right)}
$$

in which, as usual, standard Newtonian physics is assumed, and the computed one by including the Yukawa term. Notice that $A_{\text {terr }}\left(R_{\mathrm{e}}\right)$ denotes the acceleration of gravity measured on the Earth's surface with ground-based techniques, while $A_{\mathrm{L}}\left(R_{\mathrm{e}}\right)$ is a shorthand for the ratio of $\mu_{\text {meas }}^{(\mathrm{SLR})}$, i.e. the Earth's gravitational parameter empirically determined with the SLR observations to LAGEOS, to the square of the Earth's radius. When $\eta_{\mathrm{Y}}$ is computed, $A_{\mathrm{L}}\left(R_{\mathrm{e}}\right)$ is replaced by the product of the Newtonian+Yukawa acceleration $A_{\mathrm{N}+\mathrm{Y}}\left(r_{\mathrm{L}}\right)$ evaluated at distance $r_{\mathrm{L}}$ times $\left(r_{\mathrm{L}} / R_{\mathrm{e}}\right)^{2}$, while $A_{\text {terr }}\left(R_{\mathrm{e}}\right)$ is replaced by the Newtonian+Yukawa acceleration evaluated on the Earth's surface $A_{\mathrm{N}+\mathrm{Y}}\left(R_{\mathrm{e}}\right)$. Also $\eta_{\mathrm{Y}}$ is independent, by construction, of $\mu_{\infty}$. Instead, 36] insert $\mu_{\text {meas }}^{(\mathrm{SLR})} / R_{\mathrm{e}}^{2}$ in the denominator of $\eta_{\mathrm{Y}}$ instead of posing $A_{\mathrm{N}+\mathrm{Y}}\left(r_{\mathrm{L}}\right)\left(r_{\mathrm{L}} / R_{\mathrm{e}}\right)^{2}$ : this is not consistent with all the previously followed line of reasoning, also because in such a way $\mu_{\infty}$ would not be cancelled in $\eta_{\mathrm{Y}}$. Basically, $\eta$ consists of the difference between the accelerations of gravity at the same distance, i.e. on the Earth's surface, measured with different techniques normalized to the value at the same distance obtained with one of such two techniques; again, in Newtonian physics it is expected to be zero, contrary to the Yukawa-type case. Note that in $\eta$ the value obtained from SLR is extrapolated to the Earth's surface by means of the multiplicative scaling factor $\left(r_{\mathrm{L}} / R_{\mathrm{e}}\right)^{2}$.

Notice that both for $\eta^{\prime}$ and $\eta$ the scale length $\lambda$ has been kept fixed.

\subsection{The perigee of LAGEOS II}

Actually, tighter constraints can be obtained by using the perigee of LAGEOS II in connection with eq. (16) and eq. (17). The semi-major axis of LAGEOS II is $a=12,163 \mathrm{~km}=1.2163 \times$ $10^{7} \mathrm{~m}=1.9 R_{\mathrm{e}}$, while its eccentricity is $e=0.014$.

It is well known that the perigees of the LAGEOS-type satellites are particularly sensitive to a host of non-gravitational perturbations which are, thus, a major limiting factor in constraining $\alpha$. As pointed out by Ries et al. 59] in the context of the re-analysis of the earlier tests of the general relativistic Lense-Thirring effect with the LAGEOS and LAGEOS II satellites [60], the realistic accuracy in determining the secular perigee precession of LAGEOS II is larger than the gravitomagnetic effect itself mainly because of the non-gravitational perturbations affecting such an orbital element. From Table 9.7 of [61] it is possible to infer an uncertainty of about 125 milliarcseconds per year (mas $\mathrm{yr}^{-1}$ in the following).

Another important source of systematic uncertainty is the mismodeling in the multipoles of the expansion of the non-spherical part of the gravitational potential. In particular, the fully normalized even $(\ell=2,4, \ldots)$ zonal $(m=0)$ Stokes coefficients $\bar{C}_{\ell, 0}$ cause secular perigee precessions which acts as superimposed bias. Assessing correctly their mismodeling is crucial for a realistic evaluation of the overall uncertainty affecting $\alpha$.

There are nowadays several institutions worldwide which almost routinely produce global gravity field solutions by analyzing increasing data sets from the dedicated spacecraft-based missions like CHAMP8, GRACE, GOCE, along with observations collected from many geodetic satellites of LAGEOS-type tracked by the International Laser Ranging Service 62]. Among

\footnotetext{
${ }^{7}$ For preliminary investigations on the possibility of using the perigee of LAGEOS II to constrain Yukawa-like deviations from the Newtonian inverse square law of gravity, see [38, 40]. For earlier investigations on the effect of a Yukawa-like fifth force on the perigee of LAGEOS-type satellites, see [58].

${ }^{8}$ It re-entered Earth's atmosphere on September 20, 2010.
} 
their products, collected by the International Centre for Global Earth Models (ICGEM) 63, the coefficients $\bar{C}_{\ell, m}, \bar{S}_{\ell, m}$ of the geopotential stand out.

For our purposes, it is fundamental to realistically evaluate the uncertainties in the zonals, with particular regard to $\bar{C}_{2,0}$ since it induces the largest aliasing secular perigee precession. To this aim, we will follow the approach recently described by Wagner and McAdoo [64. They felt the need of comparing not only solutions releasing the mere statistical, formal errors for the geopotential coefficients, but also older models yielding calibrated errors, by explicitly requiring that the benchmark model must be formally far more accurate than the one to be tested. Furthermore, Wagner and McAdoo 64 pointed out that their method could well be applied even to solutions not displaying formal errors at all. Wagner and McAdoo 64 remarked that the calibration of the errors in a given test model should be made by using reference solutions obtained independently: for example, a GRACE-based solution should be compared with, say, a GOCE-based solution, as done by Wagner and MacAdoo themselves 64. Even in such a case, care should be taken to avoid that the reference model adopted was not used as a-priori background model in producing the models to be tested [64].

Here we apply the method by Wagner and McAdoo [64] by choosing the CHAMP-based solution AIUB-CHAMP03S [65], the GRACE/GOCE/CHAMP/SLR-based solution GOCO2S [66] and the GOCE-only solution GOCONS [67] as test models, while we take the wholly independent GRACE/GOCE/LAGEOS-based solution EIGEN-6 [68] as formally superior reference model. EIGEN-6 was released by the GeoForschungsZentrum (GFZ), AIUB-CHAMP03S was produced by the Astronomisches Institut, Universität Bern (AIUB), while GOCO2S and GOCONS were released by the Gravity Observation COmbination (GOCO) consortium. The background gravity models adopted for AIUB-CHAMP03S were the pre-CHAMP/GRACE/GOCE JGM3 [69] and EGM96 [70] SLR-only solutions, while EIGEN-6 used 6.5 years of LAGEOS data from the time span 1 Jan 2003 till 30 June 2009. GOCO2S used the GRACE-only solution ITG-Grace2010s [71] as background model. GOCONS did not adopt any background model, and its data from GOCE cover the period 1 November 2009-17 April 2011. See the discussion in 64] about the risk of precluding an unbiased calibration employing an external standard model. As requested by Wagner and McAdoo [64], the formal $\sigma_{\bar{C}_{2,0}}$ of EIGEN-6 is smaller than those of the models to be tested by about 1 order of magnitude. Instead of using a single coefficient

Table 1: First row: uncertainty $\delta \bar{C}_{2,0}$ in the even zonal of degree $\ell=2$ evaluated according to the method by Wagner and McAdoo [64]. EIGEN-6 68] was assumed as formally superior reference model $\left(\sigma_{\bar{C}_{2,0}}=2 \times 10^{-13}\right)$, while the solutions AIUB-CHAMP03S 65], GOCONS [67, and GOCO2S [66] were taken as test models. The tide system of all the models considered is tide-free. Second row: formal $\sigma_{\bar{C}_{2,0}}$ of the aforementioned models, and calibrated $\sigma_{\bar{C}_{2,0}}$ of GIF48, whose tide system is zero-tide.

\begin{tabular}{lllll}
\hline & AIUB-CHAMP03S (test) & GOCONS (test) & GOCO2S (test) & GIF48 \\
\hline$\delta \bar{C}_{2,0}$ & $3.3 \times 10^{-11}$ & $1.09 \times 10^{-10}$ & $1.08 \times 10^{-10}$ & - \\
$\sigma_{\bar{C}_{2,0}}$ & $1.1 \times 10^{-11}$ & $1.9 \times 10^{-11}$ & $4 \times 10^{-13}$ & $7.0 \times 10^{-11}$ \\
\hline
\end{tabular}

error scaling factor for the formal sigmas $\sigma_{\bar{C}_{\ell, 0}^{\text {test }}}$ of each of the test models, Wagner and McAdoo 64] also proposed to average the individual error factors over all the $2 \ell+1$ coefficients of degree 


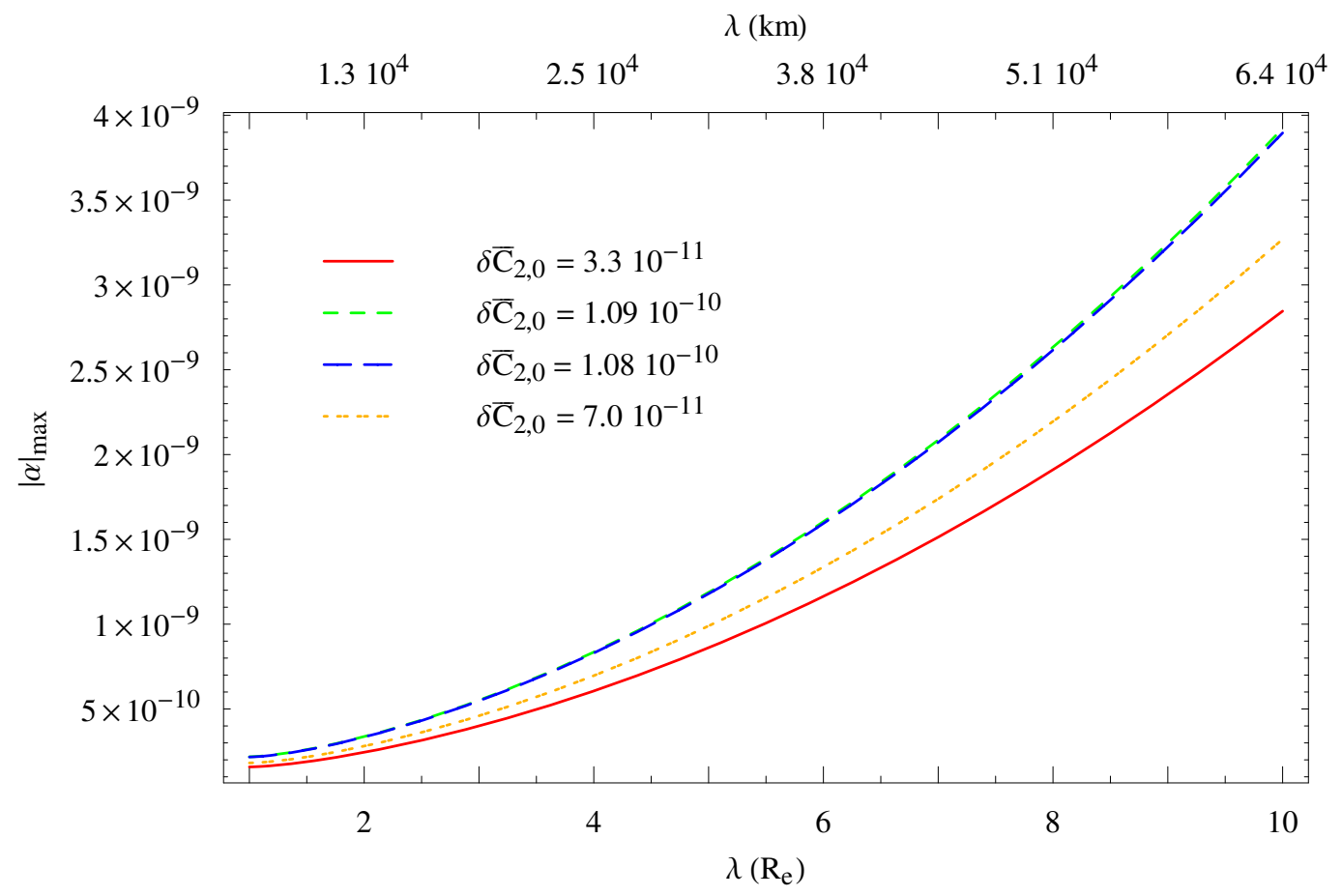

Figure 1: Upper bound on $|\alpha|$ for the Earth-LAGEOS II system from the uncertainty in the precession of the perigee $\omega$ of LAGEOS II as a function of $\lambda$ for $1 R_{\mathrm{e}} \leq \lambda \leq 10 R_{\mathrm{e}}$. As far as the mismodeling $\delta \bar{C}_{2,0}$ in the first even zonal harmonic of the Earth's geopotential is concerned, we adopted the figures of Table 1 for it, while the uncertainty due to the non-gravitational

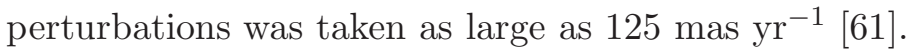

$\ell$. According to eq. (A13) of [64, one has

$$
\bar{f}_{\text {test }, \ell}=\left\{\left(\frac{1}{2 \ell+1}\right) \sum_{m=0}^{2 \ell+1}\left[\frac{\left(\bar{H}_{\ell, m}^{\mathrm{test}}-\bar{H}_{\ell, m}^{\mathrm{ref}}\right)^{2}-\left(\sigma_{\bar{H}_{\ell, m}}^{\mathrm{ref}}\right)^{2}}{\left(\sigma_{\bar{H}_{\ell, m}}^{\mathrm{test}}\right)^{2}}\right]\right\}^{\frac{1}{2}},
$$

where $\bar{H}_{\ell, m}$ denotes both $\bar{C}_{\ell, m}$ and $\bar{S}_{\ell, m}$ in the sense that the sum in eq. (21) includes all the geopotential coefficients of both kinds for a given degree $\ell$. Our results are displayed in Table 1. It can be noticed that the resulting uncertainties $\delta \bar{C}_{\ell, 0}$ are up to $1-3$ orders of magnitude larger than the formal errors. Incidentally, they are rather close to the calibrated error released in the GRACE-only GIF48 model by CSR $\left(\delta \bar{C}_{2,0}=7.0 \times 10^{-11}\right)$.

We will adopt our results in Table 1 to assess the zonals-induced bias on the Yukawainduced perigee orbital precession of LAGEOS II. Thus, by using such a figure in eq. (17), it is possible to plot the upper bound on $\alpha$ as a function of $\lambda$ : see Figure 1. It can be noted that there is an improvement of $2-5$ orders of magnitude with respect to Figure 1 of [55], Figure 1 of [35], and Figure 4 of [39]; see also Figure 1 of [57]. Lucchesi and Peron [72], using the approximate analytical results by [40] and a data record $13 \mathrm{yr}$ long for LAGEOS II, claim $|\alpha| \leq 9.9 \times 10^{-12}$ at $\lambda=1 R_{\mathrm{e}}$. However, such a figure should be considered just as a preliminary result coming from the statistical errors of the linear fitting of the post-fit numerically integrated 


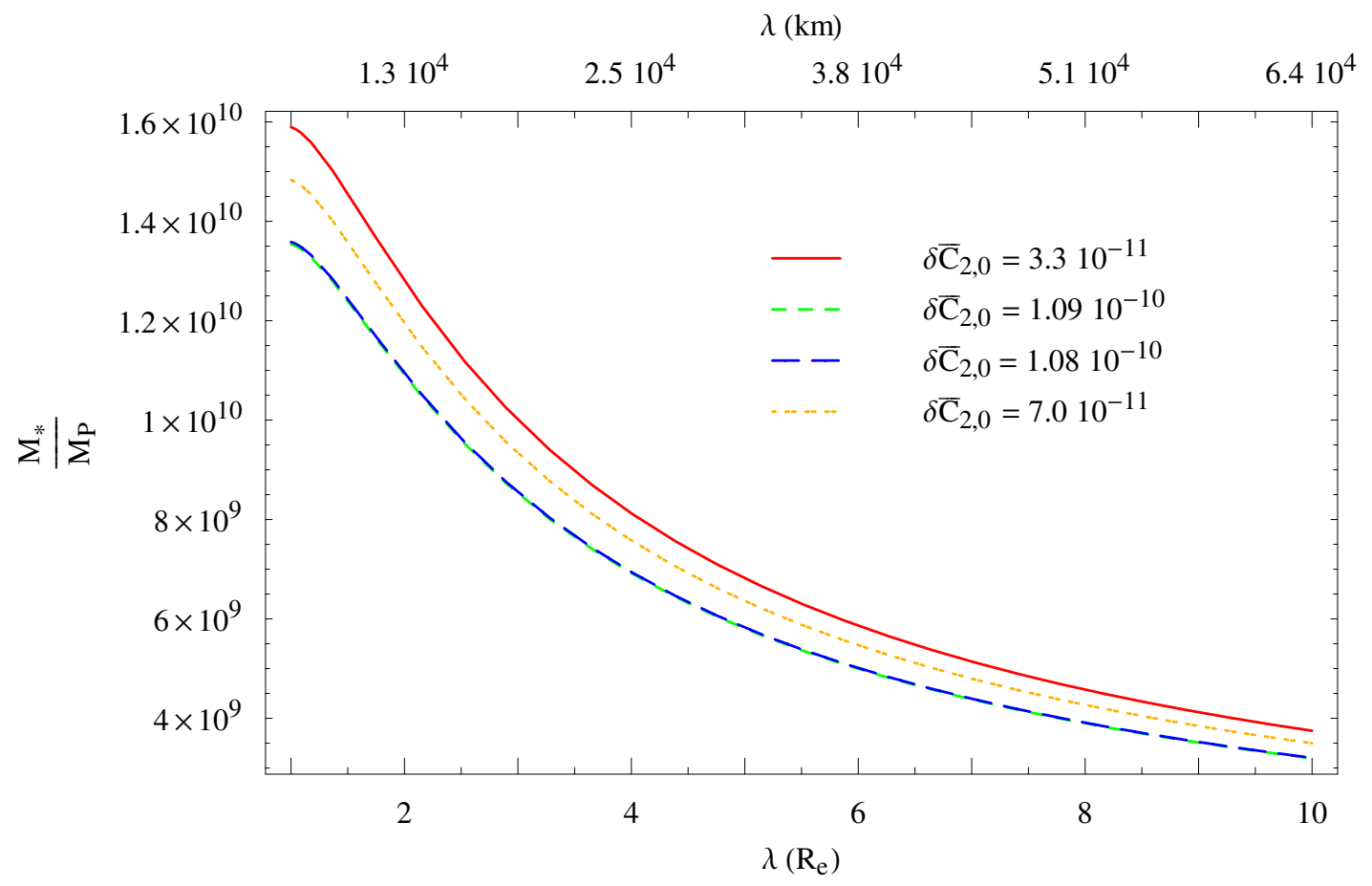

Figure 2: Lower limits on $M_{*} / M_{\mathrm{P}}$ from eq. (8) for $\xi \lesssim 0.5 \times 10^{-5}$ [1, 9] and the bounds on $\alpha$ of Figure 1 .

residuals of the perigee since, as pointed out by Lucchesi and Peron themselves [72, it does not include an analysis of the systematic errors due to the uncertainties in the zonals' geopotential. Lucchesi and Peron [72] used the GRACE-only model EIGEN-GRACE02S [73], which released a calibrated $\sigma_{\bar{C}_{2,0}}=5.304 \times 10^{-11}$. Moreover, Lucchesi and Peron [72] did not explicitly include a Yukawa-like fifth force in the dynamical models fitted to the data of LAGEOS II, by assuming that it would entirely be accounted for by their numerically integrated residuals of the perigee. A similar approach with the LAGEOS satellite was followed by March et al. [74] in constraining other non-Newtonian putative effects.

The bounds on $\alpha$ of Figure 1 yield corresponding constraints on $M_{*} / M_{\mathrm{P}}$ according to eq. (8). They are depicted in Figure 2, For $\lambda=1-1.5 R_{\mathrm{e}}$, it is $M_{*} / M_{\mathrm{P}} \gtrsim 1.4-1.6 \times 10^{10}$, while for $\lambda=10 R_{\mathrm{e}}$ we have $M_{*} / M_{\mathrm{P}} \gtrsim 4-5 \times 10^{9}$. Such results are in striking contrast with $M_{*} \sim 10^{-6} M_{\mathrm{P}}$ inferred by Dvali and Vikman [17] for $\lambda=1 R_{\mathrm{e}}$.

\subsection{The perigee of the Moon}

Let us, now, consider the range $10-100 R_{\mathrm{e}}$ corresponding to $6.4 \times 10^{7}-6.4 \times 10^{8} \mathrm{~m}$; the lunar semi-major axis is $a_{\mathrm{M}}=60 R_{\mathrm{e}}=3.8 \times 10^{8} \mathrm{~m}$. To this aim, the motion of the Moon is best suited to yield tight constraints.

Reasoning in term of the lunar perigee, the uncertainty in determining its secular precession is of the order of $\delta \dot{\varpi} \approx 0.1$ mas yr$^{-1}[75,76,77,78,79]$. The resulting bounds on $\alpha$ are depicted in Figure 3, It can be noted that $|\alpha| \lesssim 3 \times 10^{-11}$ for $\lambda=60 R_{\mathrm{e}}=3.8 \times 10^{8} \mathrm{~m}$, in substantial agreement with Figure 1 of [57, Figure 1 of [55] and Figure 4 of [39]. Cfr. also with the upper 


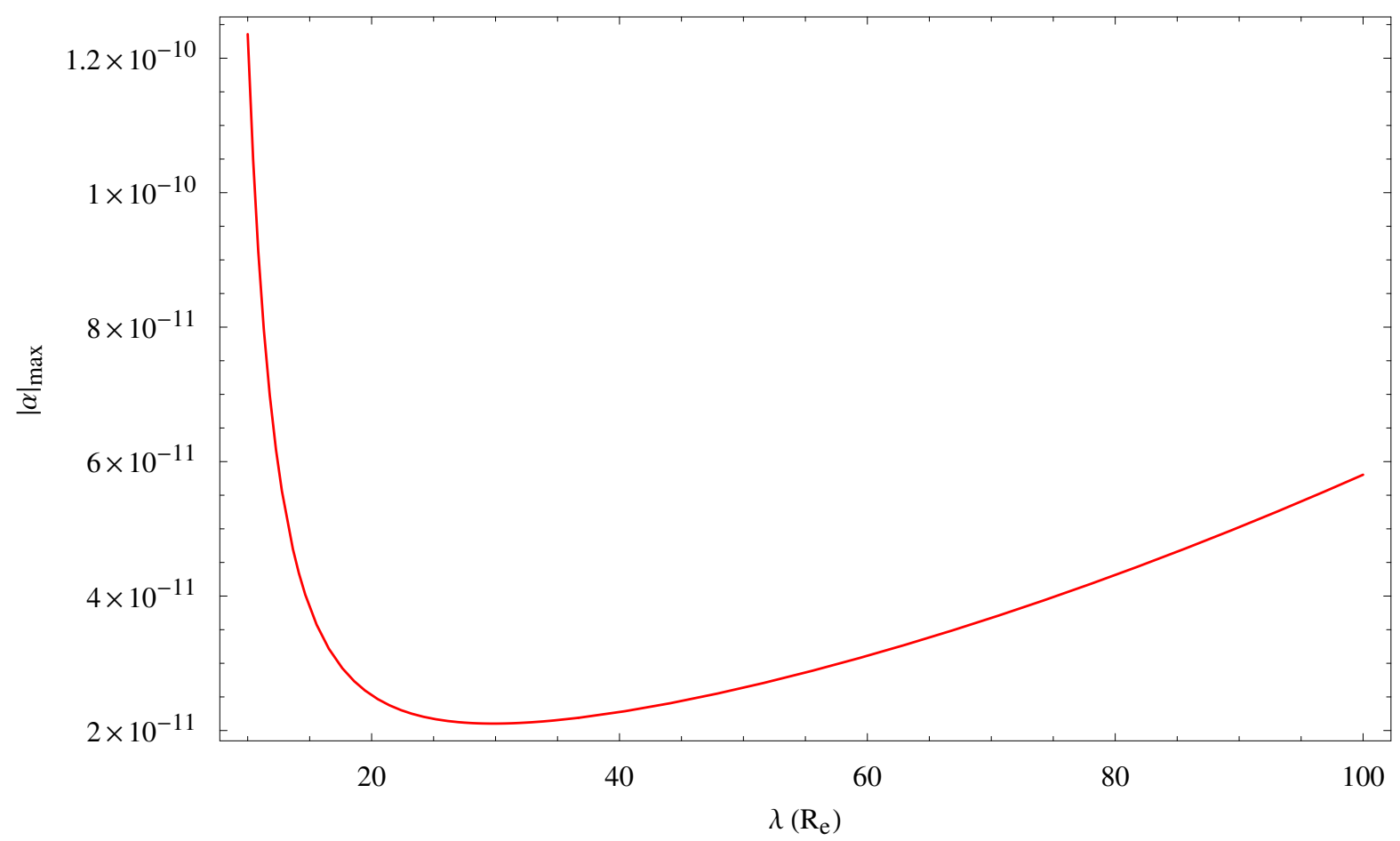

Figure 3: Upper bound on $\alpha$ for the Earth-Moon system from the uncertainty in determining the precession of the perigee of the Moon, assumed as large as $0.1{\operatorname{mas~} \mathrm{yr}^{-1}}_{\text {[75, }}$ 76, 177, 78, 779], as a function of $\lambda$ for $10 R_{\mathrm{e}} \leq \lambda \leq 100 R_{\mathrm{e}}$. 


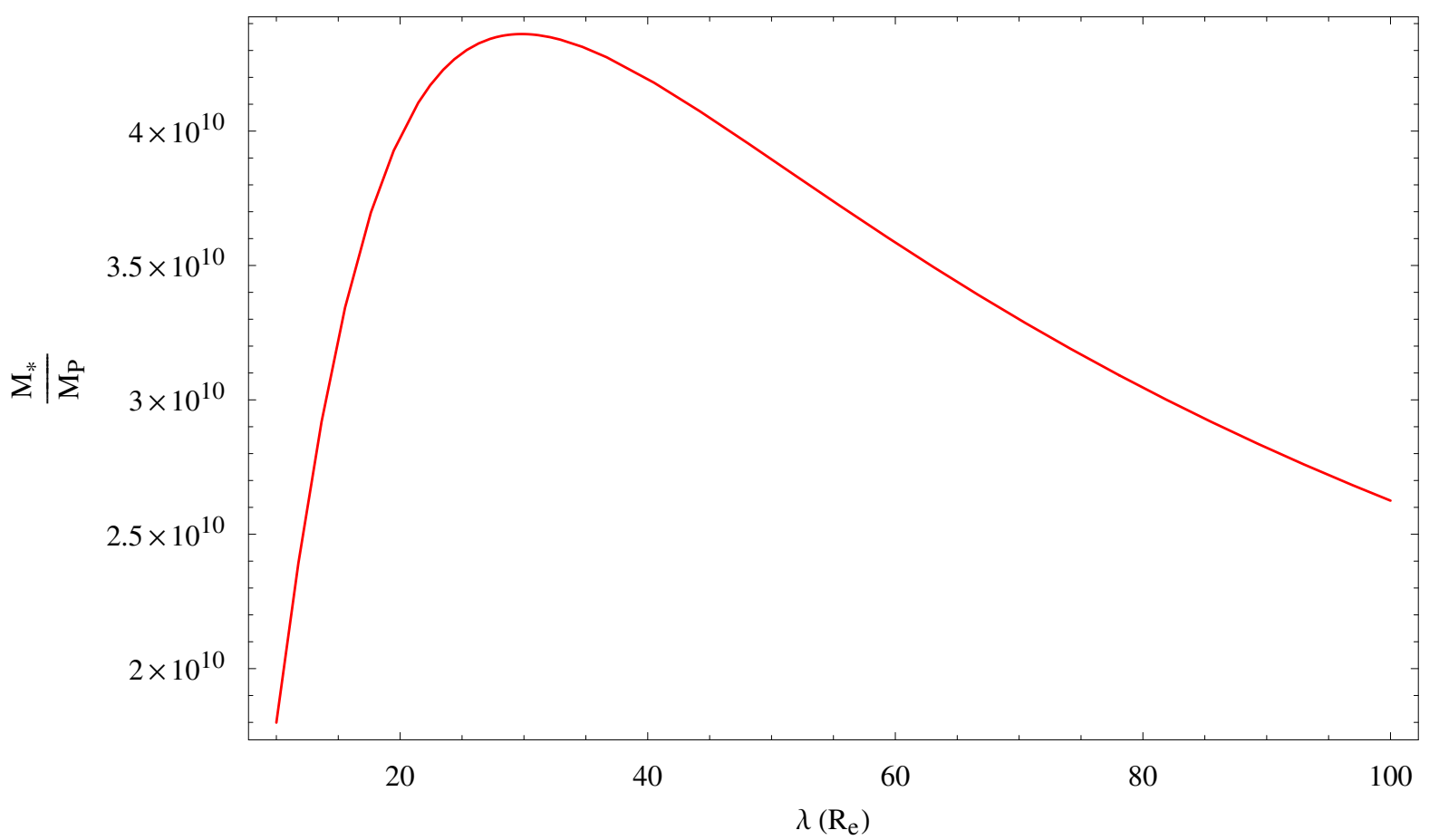

Figure 4: Lower bounds on $M_{*} / M_{\mathrm{P}}$ from eq. (8) for $\xi \lesssim 0.5 \times 10^{-5}$ [1] and the lunar bounds on $\alpha$ of Figure 3 .

bound of $|\alpha| \leq 5 \times 10^{-11}\left(\lambda=60 R_{\mathrm{e}}\right)$ by 9 Müller et al. [78, 79]. However, the constraints of Figure 3 are orders of magnitude better than those reported in [57, 55, 39] for $\lambda \neq 60 R_{\mathrm{e}}$. We also note that 10 [80] obtained $|\alpha| \lesssim 10^{-8}$ for $1.8 R_{\mathrm{e}} \lesssim \lambda \lesssim 60 R_{\mathrm{e}}$.

In Figure 4 we display the corresponding bounds on $M_{*} / M_{\mathrm{P}}$. While for $\lambda=10 R_{\mathrm{e}}$ it is $M_{*} / M_{\mathrm{P}} \sim 2 \times 10^{10}$, we have $M_{*} / M_{\mathrm{P}} \gtrsim 3.5 \times 10^{10}$ at $\lambda \sim 60 R_{\mathrm{e}}$, with $M_{*} / M_{\mathrm{P}} \gtrsim 4.5 \times 10^{10}$ at $\lambda \sim 30 R_{\mathrm{e}}$.

Finally, we remark that using data from the Lunar Reconnaissance Orbiter (LRO) should allow to further improve the bounds on $|\alpha|$ at $\lambda=60 R_{\mathrm{e}}[81]$.

\section{Discussions and conclusions}

We analytically worked out some orbital effects induced by a hypothetical gravitational fifth force of Yukawa-type on the orbital motion of a test particle moving around a central body acting as source of the modified gravitational field. We restricted ourselves neither to any specific size for the scale length parameter $\lambda$ nor to small values of the orbital eccentricity $e$ of the particle. We obtained secular precessions for the pericenter $\omega$. Our results imply the use of the modified Bessel functions of the first kind $I_{k}(x), k=0,1$.

We used the Satellite Laser Ranging data of the artificial satellite LAGEOS II to put

\footnotetext{
${ }^{9}$ Müller et al. [78, 79] actually included a Yukawa-type fifth force in the mathematical force models with which they analyzed the LLR data.

${ }^{10}$ I thank W.-T. Ni for having pointed out it to me.
} 
constraints on the coupling strength parameter $\alpha$ of the putative fifth force for the EarthLAGEOS II system in the range $1 R_{\mathrm{e}} \leq \lambda \leq 10 R_{\mathrm{e}}$ obtaining $1 \times 10^{-10} \lesssim|\alpha|_{\max } \lesssim 4 \times 10^{-9}$. The accuracy in determining the Moon's perigee from the Lunar Laser Ranging technique allowed us to infer $|\alpha|_{\max } \sim 3 \times 10^{-11}$ for the Earth-Moon pair in the range $15 R_{\mathrm{e}} \lesssim \lambda \lesssim 60 R_{\mathrm{e}}$. From $M_{*} / M_{\mathrm{P}} \sim 1 / \xi \sqrt{\alpha}$ and by using $\xi \lesssim 0.5 \times 10^{-5}$, coming from the overall uncertainty in the neutrinic time-of-flight measurements common to both OPERA and ICARUS collaborations, our bounds on $\alpha$ correspond to $M_{*} / M_{\mathrm{P}} \gtrsim 4 \times 10^{9}-1.6 \times 10^{10}$ for $1 R_{\mathrm{e}} \leq \lambda \leq 10 R_{\mathrm{e}}$, and to $M_{*} / M_{\mathrm{P}} \gtrsim 3 \times 10^{10}-4.5 \times 10^{10}$ for $15 R_{\mathrm{e}} \lesssim \lambda \lesssim 60 R_{\mathrm{e}}$, with the maximum value occurring at $\lambda \sim 30 R_{\mathrm{e}}$. Such bounds on $M_{*}$ disagree with $M_{*} \sim 10^{-6} M_{\mathrm{P}}$ by Dvali and Vikman.

Our results are neither necessarily limited to the OPERA neutrino scenario nor to the Dvali-Vikman model, being valid for any theoretical scenario yielding an effective long-range Yukawa-type correction to the Newtonian inverse-square law.

As a final, technical remark, we notice that a complementary treatment would require that a Yukawa-type extra-force should be explicitly modeled in the softwares used to process the data records, and a dedicated solve-or parameter should be estimated in dedicated data reduction procedures in which the ad-hoc modified dynamical models are fitted to the existing data sets. Such an approach has not yet been implemented in some of the analyses previously described like the LAGEOS II one: it is a drawback common to the LAGEOS-based Lense-Thirring analyses implemented so far.

\section{Acknowledgments}

I thank G. Dvali, R. Konoplya and W.-T. Ni for insightful correspondence. I am also grateful to M. Schreck for having disclosed some typos.

\section{References}

[1] T. Adam, N. Agafonova, A. Aleksandrov, O. Altinok, P. Alvarez Sanchez, A. Anokhina, S. Aoki, A. Ariga, T. Ariga, D. Autiero, A. Badertscher, A. Ben Dhahbi, A. Bertolin, C. Bozza, T. Brugière, R. Brugnera, F. Brunet, G. Brunetti, S. Buontempo, B. Carlus, F. Cavanna, A. Cazes, L. Chaussard, M. Chernyavsky, V. Chiarella, A. Chukanov, G. Colosimo, M. Crespi, N. D'Ambrosio, G. De Lellis, M. De Serio, Y. Dclais, P. del Amo Sanchez, F. Di Capua, A. Di Crescenzo, D. Di Ferdinando, N. Di Marco, S. Dmitrievsky, M. Dracos, D. Duchesneau, S. Dusini, J. Ebert, I. Efthymiopoulos, O. Egorov, A. Ereditato, L. S. Esposito, J. Favier, T. Ferber, R. A. Fini, T. Fukuda, A. Garfagnini, G. Giacomelli, M. Giorgini, M. Giovannozzi, C. Girerd, J. Goldberg, C. Gllnitz, D. Golubkov, L. Goncharov, Y. Gornushkin, G. Grella, F. Grianti, E. Gschwendtner, C. Guerin, A. M. Guler, C. Gustavino, C. Hagner, K. Hamada, T. Hara, M. Hierholzer, A. Hollnagel, M. Ieva, H. Ishida, K. Ishiguro, K. Jakovcic, C. Jollet, M. Jones, F. Juget, M. Kamiscioglu, J. Kawada, S. H. Kim, M. Kimura, E. Kiritsis, N. Kitagawa, B. Klicek, J. Knuesel, K. Kodama, M. Komatsu, U. Kose, I. Kreslo, C. Lazzaro, J. Lenkeit, A. Ljubicic, A. Longhin, A. Malgin, G. Mandrioli, J. Marteau, T. Matsuo, N. Mauri, A. Mazzoni, E. Medinaceli, F. Meisel, A. Meregaglia, P. Migliozzi, S. Mikado, D. Missiaen, K. Morishima, U. Moser, M. T. Muciaccia, N. Naganawa, T. Naka, M. Nakamura, T. Nakano, Y. Nakatsuka, V. Nikitina, F. Nitti, S. Ogawa, N. Okateva, A. Olchevsky, O. Palamara, A. Paoloni, B. D. Park, I. G. Park, A. Pastore, L. Patrizii, E. Pennacchio, H. Pessard, C. Pistillo, N. Polukhina, M. Pozzato, 
K. Pretzl, F. Pupilli, R. Rescigno, F. Riguzzi, T. Roganova, H. Rokujo, G. Rosa, I. Rostovtseva, A. Rubbia, A. Russo, O. Sato, Y. Sato, J. Schuler, L. Scotto Lavina, J. Serrano, A. Sheshukov, H. Shibuya, G. Shoziyoev, S. Simone, M. Sioli, C. Sirignano, G. Sirri, J. S. Song, M. Spinetti, L. Stanco, N. Starkov, S. Stellacci, M. Stipcevic, T. Strauss, S. Takahashi, M. Tenti, F. Terranova, I. Tezuka, V. Tioukov, P. Tolun, N. T. Tran, S. Tufanli, P. Vilain, M. Vladimirov, L. Votano, J.-L. Vuilleumier, G. Wilquet, B. Wonsak, J. Wurtz, C. S. Yoon, J. Yoshida, Y. Zaitsev, S. Zemskova, and A. Zghiche, "Measurement of the neutrino velocity with the opera detector in the cngs beam," arXiv:1109.4897 [hep-ex].

[2] G. Brumfiel, "Particles break light-speed limit," Nature (22 september, 2011) . http://www.nature.com/news/2011/110922/full/news.2011.554.html.

[3] A. Cho, "Neutrinos travel faster than light, according to one experiment," Science (22 septmber, 2011) . http://news.sciencemag.org/sciencenow/2011/09/neutrinos-travel-faster-than-lig.html.

[4] L. Grossmann, "Dimension-hop may allow neutrinos to cheat light speed," New Scientist (23 september, 2011) . http://www.newscientist.com/article/dn20957-dimensionhop-may-allow-neutrinos-to-cheat-light-speed.htn

[5] L. Grossmann, "Faster-than-light neutrino claim bolstered," New Scientist (23 september, 2011) . http://www.newscientist.com/article/dn20961-fasterthanlight-neutrino-claim-bolstered.html.

[6] E. Reich, "Speedy neutrinos challenge physicists," Nature 477 (27 september, 2011) 520. http://www.nature.com/news/2011/110927/full/477520a.html.

[7] E. Cartlidge, "Breaking news: Error undoes faster-than-light neutrino results," ScienceInsider (22 february, 2012) . http://news.sciencemag.org/scienceinsider/2012/02/breaking-news-error-undoes-faster.html?ref=hp.

[8] E. Cartlidge, "Breaking news: Official word on superluminal neutrinos leaves warp-drive fans a shred of hopebarely," ScienceInsider (24 february, 2012) . http://news.sciencemag.org/scienceinsider/2012/02/official-word-on-superluminal-ne.html?ref=hp.

[9] M. Antonello, P. Aprili, B. Baibussinov, M. Baldo Ceolin, P. Benetti, E. Calligarich, N. Canci, F. Carbonara, S. Centro, A. Cesana, K. Cieslik, D. B. Cline, A. G. Cocco, A. Dabrowska, D. Dequal, A. Dermenev, R. Dolfini, C. Farnese, A. Fava, A. Ferrari, G. Fiorillo, D. Gibin, A. Gigli Berzolari, S. Gninenko, A. Guglielmi, M. Haranczyk, J. Holeczek, A. Ivashkin, J. Kisiel, I. Kochanek, J. Lagoda, S. Mania, G. Mannocchi, A. Menegolli, G. Meng, C. Montanari, S. Otwinowski, L. Periale, A. Piazzoli, P. Picchi, F. Pietropaolo, P. Plonski, A. Rappoldi, G. L. Raselli, M. Rossella, C. Rubbia, P. Sala, E. Scantamburlo, A. Scaramelli, E. Segreto, F. Sergiampietri, D. Stefan, J. Stepaniak, R. Sulej, M. Szarska, M. Terrani, F. Varanini, S. Ventura, C. Vignoli, H. Wang, X. Yang, A. Zalewska, and K. Zaremba, "Measurement of the neutrino velocity with the icarus detector at the cngs beam," arXiv:1203.3433 [hep-ex].

[10] E. Recami, Annuario della EST. Enciclopedia della Scienza e della Tecnica, vol. 73, ch. I tachioni, pp. 85-94. Mondadori, Milan, 1973. 
[11] E. Giannetto, G. Maccarrone, R. Mignani, and E. Recami, "Are muon neutrinos faster-than-light particles?," Physics Letters B 178 no. 1, (1986) 115-120.

[12] J. Alfaro, "Quantum gravity and lorentz invariance violation in the standard model," Physical Review Letters 94 no. 22, (2005) 221302.

[13] V. Gharibyan, "Possible observation of photon speed energy dependence," Physics Letters B 611 no. 3-4, (2005) 231-238.

[14] H. Päs, S. Pakvasa, and T. Weiler, "Sterile-active neutrino oscillations and shortcuts in the extra dimension," Physical Review D 72 no. 9, (2005) 095017.

[15] J. Dent, H. Päs, S. Pakvasa, and T. Weiler, "Neutrino time travel," in Proceedings of The 15th international Conference on Supersymmetry and the Unification of fundamental Interactions SUSY 2007 July 26-August 1, 2007 Karlsruhe, Germany, W. de Boer and I. Gebauer, eds., p. 760. Brno: University of Karlsruhe in collaboration with Tribun EU s. r. o., 2008.

[16] S. Hollenberg, O. Micu, H. Päs, and T. Weiler, "Baseline-dependent neutrino oscillations with extra-dimensional shortcuts," Physical Review D 80 no. 9, (2009) 093005.

[17] G. Dvali and A. Vikman, "Price for environmental neutrino-superluminality," Journal of High Energy Physics 02 (2012) 134.

[18] G. Dvali, September, 2011. Private communication to the author.

[19] M. Anacleto, F. Brito, and E. Passos, "Supersonic velocities in noncommutative acoustic black holes," Physical Review D 85 no. 2, (2012) 025013.

[20] X.-J. Bi, P.-F. Yin, Z.-H. Yu, and Q. Yuan, "Constraints and tests of the opera superluminal neutrinos," Physical Review Letters 107 no. 24, (2011) 241802.

[21] E. Ciuffoli, J. Evslin, J. Liu, and Z. X., "Opera and a neutrino dark energy model," arXiv:1109.6641 [hep-ph].

[22] N. Itoh, H. Hayashi, A. Nishikawa, and Y. Kohyama, "Neutrino energy loss in stellar interiors. vii. pair, photo-, plasma, bremsstrahlung, and recombination neutrino processes," The Astrophysics Journal Supplement 102 (February, 1996) 411-424.

[23] A. Kehagias, "Relativistic superluminal neutrinos," arXiv:1109.6312 [hep-ph].

[24] R. Konoplya, "Superluminal neutrinos and the tachyon's stability in the rotating universe," Physics Letters B 706 no. 4, (2012) 451-455.

[25] G. Kraniotis, "Exact deflection of a neutral-tachyon in the kerrs gravitational field," arXiv:1110.1223 [gr-qc].

[26] D. Lüst and M. Petropoulos, "Comment on superluminality in general relativity," arXiv:1110.0813 [gr-qc].

[27] C. Pfeifer and M. Wohlfarth, "Beyond the speed of light on finsler spacetimes," arXiv:1109.6005 [gr-qc]. 
[28] E. Saridakis, "Superluminal neutrinos in horava-lifshitz gravity," arXiv:1110.0697 [gr-qc].

[29] S. Vacaru, "Super-luminal effects for finsler branes as a way to preserve the paradigm of relativity theories," arXiv:1110.0675 [physics.gen-ph].

[30] P. Wang, H. Wu, and H. Yang, "Superluminal neutrinos and domain walls," arXiv:1109.6930 [hep-ph].

[31] M. Schreck, "Multiple lorentz groups-a toy model for superluminal opera neutrinos," arXiv:1111.7268 [hep-ph].

[32] G. Dvali, G. Gabadadze, M. Kolanovic, and F. Nitti, "Power of brane-induced gravity," Physical Review D 64 no. 8, (2001) 084004.

[33] B. Bertotti, P. Farinella, and D. Vokrouhlický, Physics of the Solar System. Kluwer Academic Press, Dordrecht, 2003.

[34] C. Burgess and J. Cloutier, "Astrophysical evidence for a weak new force?," Physical Review D 38 no. 10, (1988) 2944.

[35] C. Talmadge, J.-P. Berthias, R. Hellings, and E. Standish, "Model-independent constraints on possible modifications of newtonian gravity," Physical Review Letters 61 no. 10, (1988) 1159-1162.

[36] E. Fischbach and C. Talmadge, The Search for Non-Newtonian Gravity. Springer-Verlag, New York, 1999.

[37] K. Nordtvedt, "Improving gravity theory tests with solar system "grand fits"," Physical Review D 61 no. 12, (2000) 122001.

[38] L. Iorio, "Constraints to a yukawa gravitational potential from laser data to lageos satellites," Physics Letters A 298 no. 5-6, (2002) 315-318.

[39] E. Adelberger, B. Heckel, and N. A.E., "Tests of the gravitational inverse-square law," Annual Review of Nuclear and Particle Science 53 (December, 2003) 77-121.

[40] D. Lucchesi, "Lageos ii perigee shift and schwarzschild gravitoelectric field," Physics Letters A 318 no. 3, (2003) 234-240.

[41] N. Kolosnitsyn and V. Melnikov, "Test of the inverse square law through precession of orbits," General Relativity and Gravitation 36 no. 7, (2004) 1619-1624.

[42] N. Kolosnitsyn and V. Melnikov, "New observational tests of non-newtonian interactions at planetary and binary pulsar orbital distances," Gravitation and Cosmology 10 no. 1, (2004) 137-140.

[43] O. Bertolami and J. Páramos, "Astrophysical constraints on scalar field models," Physical Review D 71 no. 2, (2005) 023521.

[44] S. Reynaud and M.-T. Jaekel, "Testing the newton law at long distances," International Journal of Modern Physics A 20 no. 11, (2005) 2294-2303. 
[45] M. Sereno and P. Jetzer, "Dark matter versus modifications of the gravitational inverse-square law: results from planetary motion in the solar system," Monthly Notices of the Royal Astronomical Society 371 no. 2, (2006) 626-632.

[46] G. Adkins and J. McDonnell, "Orbital precession due to central force perturbations," Physical Review D 75 no. 8, (2007) 082001.

[47] L. Iorio, "First preliminary tests of the general relativistic gravitomagnetic field of the sun and new constraints on a yukawa-like fifth force from planetary data," Planetary and Space Science 55 no. 10, (2007) 1290-1298.

[48] L. Iorio, "Constraints on the range $\lambda$ of yukawa-like modifications to the newtonian inverse-square law of gravitation from solar system planetary motions," Journal of High Energy Physics 10 (2007) 041.

[49] L. Iorio, "Putting yukawa-like modified gravity (mog) on the test in the solar system," Scholarly Research Exchange 2008 (2008) 238385.

[50] J. Moffat, "A modified gravity and its consequences for the solar system, astrophysics and cosmology," International Journal of Modern Physics D 16 no. 12A, (2008) 2075-2090.

[51] X.-M. Deng, Y. Xie, and T.-Y. Huang, "Modified scalar-tensor-vector gravity theory and the constraint on its parameters," Physical Review D $\mathbf{7 9}$ no. 4, (2009) 044014.

[52] I. Haranas and O. Ragos, "Yukawa-type effects in satellite dynamics," Astrophysics and Space Science 331 no. 1, (2011) 115-119.

[53] I. Haranas and O. Ragos, "Calculation of radar signal delays in the vicinity of the sun due to the contribution of a yukawa correction term in the gravitational potential," Astrophysics and Space Science 334 no. 1, (2011) 71-74.

[54] I. Haranas, O. Ragos, and M. Vasile, "Yukawa-type potential effects in the anomalistic period of celestial bodies," Astrophysics and Space Science 332 no. 1, (2011) 107-113.

[55] D. Krause and E. Fischbach, Gyros, Clocks, Interferometers.... :Testing Relativistic Gravity in Space, vol. 562 of Lecture Notes in Physics, ch. Searching for Extra Dimensions and New String-Inspired Forces in the Casimir Regime, pp. 292-309. Springer-Verlag, Berlin, 2001.

[56] J. Moffat, "Modified gravity or dark matter?," in International conference on Two Cosmological Models, Universidad Iberoamericana, Ciudad de Mexico, November 17-19, 2010. 2011. arXiv:1101.1935 [astro.ph.CO].

[57] K. Nordtvedt, Gyros, Clocks, Interferometers... :Testing Relativistic Gravity in Space, vol. 562 of Lecture Notes in Physics, ch. An Overview of Solar System Gravitational Physics: The Theory-Experiment Interface, pp. 4-14. Springer-Verlag, Berlin, 2001.

[58] K. Nordtvedt, LARES Laser Relativity Satellite for the study of the Earth gravitational field and general relativity measurements. An ASI Small Mission. Phase A Report, ch. LARES and Tests on New Long Range Forces, pp. 34-37. Università di Roma "La Sapienza", Rome, 30th october, 1998. 
[59] J. Ries, R. Eanes, and B. Tapley, Nonlinear Gravitodynamics. The Lense-Thirring Effect, ch. Lense-Thirring Precession Determination from Laser Ranging to Artificial Satellites, pp. 201-211. World Scientific, Singapore, 2003.

[60] I. Ciufolini, E. Pavlis, F. Chieppa, E. Fernandes-Vieira, and J. Pérez-Mercader, "Test of general relativity and measurement of the lense-thirring effect with two earth satellites," Science 279 no. 5359, (1998) 2100-2103.

[61] D. Lucchesi, The Measurement of Gravitomagnetism: a Challenging Enterprise, ch. The LAGEOS Satellites: Non-Gravitational Perturbations and the Lense-Thirring Effect, pp. 137-155. NOVA Science Publishers, Hauppauge (N.Y.), 2007.

[62] M. Pearlman, J. Degnan, and J. Bosworth, "The international laser ranging service," Advances in Space Research 30 no. 2, (2002) 135-143.

[63] F. Barthelmes and W. Köhler, "A web based service for using global earth gravity field models," in Arbeitskreis Geodäsie/Geophysik, Herbsttagung 2010 19-22 October 2010, Smolenice, Slovakia. Deutsches GeoForschungsZentrum GFZ, September, 2010.

[64] C. A. Wagner and D. C. McAdoo, "Error calibration of geopotential harmonics in recent and past gravitational fields," Journal of Geodesy 86 no. 2, (2012) 99-108.

[65] A. Jäggi, L. Prange, U. Meyer, L. Mervart, G. Beutler, T. Gruber, R. Dach, and R. Pail, "Gravity field determination at aiub: From annual to multi-annual solutions," in EGU General Assembly 2010, 2-7 May, 2010 in Vienna, Austria. European Geophysical Union, May, 2010. http://aiuws.unibe.ch/download/various/AIUB-CHAMP03S\$_\$120.gfc. paper number EGU2010-5842.

[66] H. Goiginger, E. Hoeck, D. Rieser, T. Mayer-Gürr, A. Maier, S. Krauss, R. Pail, T. Fecher, T. Gruber, J. Brockmann, I. Krasbutter, W.-D. Schuh, A. Jäggi, L. Prange, W. Hausleitner, O. Baur, and J. Kusche, "The satellite-only global gravity field model goco02s," in EGU General Assembly 2011, 3rd-8th April 2011, Vienna, Austria. European Geophysical Union, April, 2011. paper number EGU2011-10571.

[67] R. Pail, S. Bruinsma, F. Migliaccio, C. Foerste, H. Goiginger, W.-D. Schuh, E. Hoeck, M. Reguzzoni, J. Brockmann, O. Abrikosov, M. Veicherts, T. Fecher, R. Mayrhofer, I. Krasbutter, F. Sansò, and C. Tscherning, "First goce gravity field models derived by three different approaches," Journal of Geodesy 85 no. 11, (2011) 819-843.

[68] C. Förste, S. Bruinsma, R. Shako, J.-C. Marty, F. Flechtner, O. Abrikosov, C. Dahle, J.-M. Lemoine, H. Neumayer, R. Biancale, F. Barthelmes, R. König, , and G. Balmino, "Eigen- 6 a new combined global gravity field model including goce data from the collaboration of gfz-potsdam and grgs-toulouse," in EGU General Assembly 2011, 3rd-8th April 2011, Vienna, Austria. European Geophysical Union, April, 2011. paper number EGU2011-3242.

[69] B. Tapley, M. Watkins, J. Ries, G. Davis, R. Eanes, S. Poole, H. Rim, B. Schutz, C. Shum, R. Nerem, F. Lerch, J. Marshall, S. Klosko, N. Pavlis, and R. Williamson, "The joint gravity model 3," Journal of Geophysical Research 101 no. B12, (1996) 28029-28049. 
[70] F. Lemoine, S. Kenyon, J. Factor, R. Trimmer, N. Pavlis, D. Chinn, C. Cox, S. Klosko, S. Luthcke, M. Torrence, Y. Wang, R. Williamson, E. Pavlis, R. Rapp, and T. Olson, "The development of the joint nasa gsfc and the national imagery and mapping agency (nima) geopotential modelegm96," NASA Technical Paper NASA/TP1998206861, Goddard Space Flight Center, Greenbelt, USA, 1998.

[71] T. Mayer-Gürr, E. Kurtenbach, and A. Eicker, "Itg-grace2010." Online document, 2010. http://www.igg.uni-bonn.de/apmg/index.php?id=itg-grace2010.

[72] D. Lucchesi and R. Peron, "Accurate measurement in the field of the earth of the general-relativistic precession of the lageos ii pericenter and new constraints on non-newtonian gravity," Physical Review Letters 105 no. 23, (2010) 231103.

[73] C. Reigber, R. Schmidt, F. Flechtner, R. König, U. Meyer, K.-H. Neumayer, P. Schwintzer, and S. Y. Zhu, "An earth gravity field model complete to degree and order 150 from grace: Eigen-grace02s," Journal of Geodynamics 39 no. 1, (2005) 1-10.

[74] R. March, G. Bellettini, R. Tauraso, and S. Dell'Agnello, "Constraining spacetime torsion with lageos," General Relativity and Gravitation 43 no. 11, (2011) 3099-3126.

[75] J. Dickey, P. Bender, J. Faller, X. Newhall, R. Ricklefs, J. Ries, P. Shelus, C. Veillet, A. Whipple, J. Wiant, J. Williams, and C. Yoder, "Lunar laser ranging: A continuing legacy of the apollo program," Science 265 no. 5171, (1994) 482-490.

[76] J. Müller, M. Schneider, M. Soffel, and H. Ruder, "Testing einstein's theory of gravity by analyzing lunar laser ranging data," The Astrophysical Journal Letters 382 (December, 1991) L101-L103.

[77] J. Williams, X. Newhall, and J. Dickey, "Relativity parameters determined from lunar laser ranging," Physical Review D 53 no. 12, (1996) 6730-6739.

[78] J. Müller, J. Williams, S. Turyshev, and P. Shelus, Dynamic Planet 2005: Monitoring and Understanding a Dynamic Planet with Geodetic and Oceanographic Tools, ch. Potential capabilities of lunar laser ranging for geodesy and relativity, pp. 903-909. IAG Symposia. Springer-Verlag, Berlin, 2007.

[79] J. Müller, J. Williams, and S. Turyshev, Lasers, Clocks and Drag-Free Control: Exploration of Relativistic Gravity in Space, vol. 349 of Astrophysics and Space Science Library, ch. Lunar laser ranging contributions to relativity and geodesy, pp. 456-472. Springer-Verlag, Berlin, 2008.

[80] G. Li and H. Zhao, "Constraint on intermediate-range gravity from earth-satellite and lunar orbiter measurements and lunar laser ranging," International Journal of Modern Physics D 14 no. 10, (2005) 1657-1666.

[81] L. Tsang, "How can nasa's lunar reconnaissance orbiter projects verify the existence of the fifth force," New Astronomy 17 no. 1, (2012) 18-21. 